\title{
Frontier modes of light element isotopic substitution as a key for origin studies
}

\author{
NAOHIRO YOSHIDA ${ }^{1,2}$ \\ ${ }^{1}$ Earth-Life Science Institute, Tokyo Institute of Technology, \\ Tokyo, 152-8550, Japan, yoshida.n.aa@m.titech.ac.jp \\ ${ }^{2}$ Department of Chemical Science and Engineering, Tokyo \\ Institute of Technology, Yokohama, Kanagawa 226-8502, \\ Japan
}

There are numerous isotopic exchanged molecular species within a molecular species with complex modes such as; position specific isotope abundance (PSIA), mass independent isotope fractionation (MIF) and multiple isotope substitution (Clumped). Solving the complex modes of light element isotopic substitution of molecules of biogeochemical importance will lead to the better understanding of origin of molecules, biogeochemical processes, cycles, systems, the Earth and Life.

The cycles of trace gases and biomarker molecules have been studied. Their behaviors have been observed in space from the deep ocean to the upper atmosphere and in time from the early Earth to the present. Unique, high-dimensional, and robust isotopic methodologies have been developed and immediately applied to the biogeochemical researches.

$\mathrm{N}_{2} \mathrm{O}$ is the least understood among global warming gases, trace but ubiquitous, cycled mainly by microorganisms, and important for both global warming and ozone reduction. Nitrogen isotope ratio in bulk $\mathrm{N}_{2} \mathrm{O}$ was first studied, followed by the nitrogen PSIA, and 10 among 12 isotopomers/isotopologues can be studied at present, which has promoted the molecules better understood than before.

The number of isotopomers/isotopologues of organic molecules increases in an order of magnitude larger with addition of 1 carbon chain and the significant expansion of information is expected. The PSIA studies have been promoted; acetic acid, ethanol, pyruvate, propane, alanine, butane, vanillin, caffeine, $\mathrm{C} 6$ to $\mathrm{C} 12 \mathrm{n}$-alkane, and those from C11 to C31.

Fragmentation methodology at the ionization in or at the online pyrolysis before introduction to low-resolution and/or high-resolution isotope ratio mass spectrometer, infrared diode laser absorption spectrometry, and quantitative nuclear magnetic resonance system have been developed.

Theoretical, instrumental, simulational, and model studies have been incorporated with these parameters. These origin studies are being accelerated with some largest JSPS grantsin-aid, WPI program, and a NAI project in the midst of their 5-10 years. 